\title{
Dynamic adjustments in working memory in the face of affective interference
}

\author{
J. E. Witkin ${ }^{1}$ - A. P. Zanesco ${ }^{1}$ - E. Denkova ${ }^{1}$ - A. P. Jha ${ }^{1}$ \\ Published online: 5 August 2019 \\ (C) The Psychonomic Society, Inc. 2019
}

\begin{abstract}
Cognitive control, which allows for the selection and monitoring of goal-relevant behavior, is dynamically upregulated on the basis of moment-to-moment cognitive demands. One route by which these demands are registered by cognitive control systems is via the detection of response conflict. Yet working memory (WM) demands may similarly signal dynamic adjustments in cognitive control. In a delayed-recognition WM task, Jha and Kiyonaga (Journal of Experimental Psychology: Learning, Memory, \& Cognition, 36(4), 1036-1042, 2010) demonstrated dynamic adjustments in cognitive control via manipulations of mnemonic load and delay-spanning cognitive interference. In the present study, we aimed to extend prior work by investigating whether affective interference may similarly upregulate cognitive control. In Experiment 1, participants $(N=89)$ completed a delayed-recognition WM task in which mnemonic load (memory load of one vs. two items) and delay-spanning affective interference (neutral vs. negative distractors) were manipulated in a factorial design. Consistent with Jha and Kiyonaga, the present results revealed that mnemonic load led to dynamic adjustments in cognitive control, as reflected by greater performance on trials preceded by high than by low load. In addition, we observed that affective interference could trigger dynamic adjustments in cognitive control, as evinced by higher performance on trials preceded by negative than by neutral distractors. These findings were subsequently confirmed in Experiment 2, which was a pre-registered replication study $(N=100)$. Thus, these results suggest that in addition to dynamic adjustments as a function of mnemonic load, affective interference, similar to cognitive interference (Jha \& Kiyonaga Journal of Experimental Psychology: Learning, Memory, \& Cognition, 36(4), 1036-1042, 2010), may trigger dynamic adjustments in cognitive control during a WM task.
\end{abstract}

Keywords Working memory $\cdot$ Cognitive control $\cdot$ Emotion

\section{Background}

Successfully adapting to unpredictable and ever-changing environments is necessary in daily life. Cognitive control, which refers to a family of higher-order processes that allow for the selection, maintenance, and monitoring of behavior in the service of goal attainment, is necessary for performance success (Botvinick, Braver, Barch, Carter, \& Cohen, 2001; Braver, Gray, \& Burgess, 2007; Dreisbach \& Fischer, 2012b; Miller \& Cohen, 2001; Redick, 2014). When task demands outweigh current processing resources, upregulation of cognitive control may be triggered in the service of benefiting subsequent goal-relevant information processing. Herein, the term dynamic adjustments refers broadly to trial-by-trial

A. P. Jha

a.jha@psy.miami.edu

1 Department of Psychology, University of Miami, Coral Gables, FL, USA fluctuations in the deployment of cognitive resources dedicated to processing task-relevant information. These adjustments typically involve upregulation of cognitive control, which is characterized by increased engagement of attentional control mechanisms to facilitate subsequent goal-relevant responses (Gratton, Cooper, Fabiani, Carter, \& Karayanidis, 2018). To date, there has been a paucity of research investigating the breadth of factors capable of triggering dynamic upregulation of cognitive control.

One prominent account of the dynamic upregulation of cognitive control, proposed by Botvinick and colleagues, is the conflict-monitoring theory (Botvinick et al., 2001; Botvinick, Cohen, \& Carter, 2004; Botvinick, Nystrom, Fissell, Carter, \& Cohen, 1999). This theory suggests that ongoing mental processes are monitored for "conflict," which are instances when goal attainment may be jeopardized due to discrepancies between internal representations and prepotent behavioral tendencies. Consequently, the detection of conflict is proposed to trigger the upregulation of cognitive control processes in resolving task-related challenges. As a 
result of this upregulation, goal-relevant information processing may be facilitated in subsequent moments. The phenomenon of sequential performance benefits following conflict detection is referred to as conflict adaptation (Botvinick et al., 2001).

Studies investigating conflict adaptation have primarily utilized tasks involving response conflict, such as the Stroop, Simon, and Eriksen flanker tasks (Botvinick et al., 1999; Egner, 2007; Gratton, Coles, \& Donchin, 1992; Hommel, Proctor, \& Vu, 2004; Kerns et al., 2004; Stürmer, Leuthold, Soetens, Schröter, \& Sommer, 2002; Ullsperger, Bylsma, \& Botvinick, 2005; Verbruggen, Notebaert, Liefooghe, \& Vandierendonck, 2006). In these tasks, high-conflict trials are those in which task-irrelevant stimulus features directly interfere with response selection. In contrast, low-conflict trials have task-irrelevant stimulus features that are not in conflict with the desired response. For example, in the Stroop task, participants are asked to indicate the ink color of the presented word, which may or may not conflict with the meaning of the word (e.g., the word "Blue" presented in a green font represents a high-conflict trial, whereas the word "Blue" presented in a blue font represents a low-conflict trial). Response times (RTs) are slower and task accuracy is lower on current high- versus low-conflict trials (Botvinick et al., 1999; Gratton et al., 1992; Kerns et al., 2004; Stürmer et al., 2002). However, according to theories of conflict adaptation, highconflict trials lead to the upregulation of cognitive control to buffer against interference from task-irrelevant distracting stimuli on subsequent high-conflict trials (Botvinick et al., 2001). As such, conflict adaptation is reflected by the biasing of attention toward task-relevant information (Becker, Jostmann, \& Holland, 2019; Botvinick et al., 2001) and by facilitated performance on trials following high- versus lowconflict trials (Egner, 2007; Gratton et al., 1992; Hommel et al., 2004; Kerns et al., 2004; Stürmer et al., 2002; Ullsperger et al., 2005; Verbruggen et al., 2006).

The presence of conflict has also been conceptualized as an affectively negative experience (Botvinick, 2007; Dreisbach \& Fischer, 2012b; Fritz \& Dreisbach, 2013; Saunders, Lin, Milyavskaya, \& Inzlicht, 2017). Indeed, Dreisbach and others have proposed that the upregulation of cognitive control in response to conflict operates via the triggering of an aversive signal (see Dreisbach \& Fischer, 2012b; Saunders et al., 2017). Neuroimaging studies have supported the association between conflict signals and negative stimuli, in that both activate overlapping regions of the anterior cingulate and midcingulate cortex (ACC and MCC, respectively), and prior evidence suggests that these regions play a role in integrating conflict signals and affective information to influence cognitive control and behavior (Botvinick, 2007; Saunders et al., 2017).

A recent behavioral study investigated the link between conflict and negative affect by examining whether the presence of conflict was a better predictor of subsequent performance benefits than the subjective experience of negative affect in a response conflict task (Fröber, Stürmer, Frömer, \& Dreisbach, 2017). Participants completed a Simon task and rated their affective experience after each trial as either "pleasant" or "unpleasant." A typical conflict adaptation effect was observed, with facilitated performance following high- as compared to low-conflict trials. Strikingly, when results were analyzed as a function of the pleasantness rating, sequential performance benefits were observed following low-conflict trials rated as unpleasant but not those rated as pleasant. These findings suggest that negative affect can upregulate cognitive control, and in some cases may be enough to produce sequential performance benefits even in the absence of response conflict.

Furthermore, several studies have suggested that affectively negative stimuli embedded within response conflict tasks lead to enhanced upregulation of cognitive control. A study by Melcher, Born, and Gruber (2011) demonstrated that negative images (vs. neutral images) presented between neutral Stroop trials resulted in enhanced activation of brain areas implicated in the upregulation of cognitive control. Behaviorally, a response conflict study in which the task-relevant stimulus set included embedded task-irrelevant emotional words, revealed that negative words enhanced sequential performance relative to neutral words (Zeng et al., 2017). However, some studies have reported that negative stimuli do not trigger subsequent performance benefits more than neutral stimuli do (Dignath, Janczyk, \& Eder, 2017; van Steenbergen, Band, \& Hommel, 2009). Another study suggested that negative stimuli eliminated subsequent performance benefits (Padmala, Bauer, \& Pessoa, 2011). Thus, the evidence has been somewhat mixed regarding upregulation of cognitive control with negative (vs. neutral) stimuli in response conflict tasks.

Beyond response conflict and negatively valenced stimuli, recent studies have investigated whether subsequent performance benefits can be triggered by cognitive task demands more broadly. A study by Fischer, Dreisbach, and Goschke (2008) combined a Simon task with a secondary number comparison task. As predicted, subsequent performance benefits were observed in response to high-conflict trials in the Simon task. Surprisingly, sequential performance benefits were also observed with increased difficulty in the number comparison task. Another study by Dreisbach and Fischer (2011) examined whether cognitive demands, in the absence of conflict or response selection manipulations, resulted in subsequent performance benefits. This study used a task that varied perceptual fluency across trials by including hard-to-read and easy-to-read stimuli. The results demonstrated that hard-to-read trials (more than easy-to-read trials) facilitated performance on subsequent trials. Thus, sequential performance benefits may not be restricted to the 
presence of response conflict or negative stimuli, but may be triggered by broader demands on cognitive control.

In the present study, we examined factors that may contribute to subsequent performance benefits in the context of working memory (WM). WM is a key facet of cognitive control involving the maintenance and manipulation of information over short intervals. WM tasks typically involve a series of successive processes including encoding, maintenance, distractor interference resolution, and retrieval processes (Baddeley, 1986; Cowan, 2016). Many aspects of delayedrecognition WM tasks can be manipulated in order to vary cognitive demands. Prior studies have manipulated mnemonic load (see Jha \& McCarthy, 2000), delay-spanning interference (Dolcos, Miller, Kragel, Jha, \& McCarthy, 2007; Sreenivasan \& Jha, 2007), and retrieval demands (Cabeza, Dolcos, Graham, \& Nyberg, 2002), finding that higher demands result in poorer current-trial performance.

In a study by Jha and Kiyonaga (2010), mnemonic load and distractor interference were manipulated to investigate whether the current-trial demands impact subsequent-trial performance. At the beginning of each trial, participants were instructed to remember one or two memory item(s) (low or high load, respectively), consisting of faces or shoes. During the delay interval, participants were presented with two taskirrelevant distractors consisting of either faces or shoes that were of the same category (i.e., confusable, or high interference) or of a different category (i.e., nonconfusable, or low interference) as the memory item(s). At the end of the delay, participants were presented with a test item and asked if the test item matched or did not match the memory item. Analyses of current-trial effects demonstrated the expected effects of lower task accuracy and slower RTs for high-load than for low-load trials and for high-interference than for lowinterference trials (Jha, Fabian, \& Aguirre, 2004; Jha \& McCarthy, 2000; Sreenivasan \& Jha, 2007). An analysis of sequential performance as a function of the previous-trial demands demonstrated greater task accuracy and faster RTs for trials following high-load than for those following low-load trials, and for trials following high-interference as compared to low-interference trials. These results suggested that high load and high interference can prompt the upregulation of cognitive control in a WM task.

In the context of the delayed-recognition WM task described above, the pattern of results observed as a function of the mnemonic load manipulation was akin to the results reported by Dreisbach and Fischer (2011) for high versus low perceptual fluency, in that high versus low cognitive demands not involving conflict were able to produce subsequent performance benefits. The distractor manipulation, on the other hand, was somewhat analogous to the congruency manipulation in response conflict tasks. That is, high-interference distractor trials might have resulted in the experience of conflict, as similar-category memory items were to be maintained in WM during distractor presentation. In line with the conflict adaptation literature, high- as compared to lowinterference trials resulted in subsequent performance benefits.

Given the prior evidence that response conflict and negative affect might activate similar brain regions in order to upregulate cognitive control (Botvinick, 2007; Cohen \& Henik, 2012; Dreisbach \& Fischer, 2012a, 2015; Saunders et al., 2017), and that conflict-like interference trials result in subsequent-trial benefits, perhaps negative delay-spanning distractors might similarly result in subsequent-trial benefits during delayed-recognition WM tasks. Although previous studies have shown that negative (vs. neutral) distractors impair current-trial performance on a delayed-recognition WM task with valenced distractors (Dolcos, Diaz-Granados, Wang, \& McCarthy, 2008; Dolcos \& McCarthy, 2006; Jha, Witkin, Morrison, Rostrup, \& Stanley, 2017), these studies have not investigated subsequent-trial effects as a function of distractor valence. To test this prediction, in the present study we investigated the impact of negative and neutral delay-spanning distractors on subsequent-trial performance in a delayedrecognition WM task.

Herein, two experiments were conducted using a task similar to that of Jha and Kiyonaga (2010), in which mnemonic load and affective interference were parametrically manipulated across trials, to examine the effects of the current and previous trial demands on performance. The findings from the first experiment (Exp. 1) were used to formally pre-register a replication study (Exp. 2) using the Open Science Framework (www.osf.io). We examined the main effect of each factor (load and affective interference) on current-trial performance as a function of current and previous trial demands. For the current-trial demands, we predicted that WM performance would be greater when mnemonic load was low (one item) rather than high (two items), and affective distractors were categorized as neutral or negative on the basis of prior studies examining the effect of mnemonic load and/or affective interference in WM tasks (Dolcos et al., 2008; Dolcos \& McCarthy, 2006; Jha \& Kiyonaga, 2010; Jha et al., 2017). For previous trial demands, we predicted that WM performance would be greater on trials preceded by high- rather than low-load trials, on the basis of findings by Jha and Kiyonaga (2010), and on trials preceded by negative rather than neutral distractors, on the basis of studies suggesting that negative affect and conflict are interrelated constructs (Botvinick, 2007; Dreisbach \& Fischer, 2012b). We also examined the combined effect of previous-trial load and previous-trial affective interference on subsequent-trial performance. We predicted that performance would be highest on trials preceded by both high-demand conditions (high load and negative distractors), lower for trials preceded by one high-demand condition and one low-demand condition (high load and neutral distractors or low load and negative distractors), and 
lowest for trials preceded by both low-demand conditions (low load and neutral distractors).

\section{Experiment 1}

\section{Method}

\section{Participants}

A delayed-recognition WM task with affective distractors was administered to a group of healthy young adults recruited from the University of Miami community ( $N=89$ : 54 females, 35 males; $M$ age $=19.35$ years, $S D=1.69$ ), who received course credit for their participation. Informed consent was obtained in accordance with the Institutional Review Boards of the University of Miami.

\section{Experimental stimuli and design}

WM task with affective distractors Participants were instructed to remember an array of faces or shoes over a delay interval that included distracting images. The task was similar to those used in previous studies of WM utilizing undergraduate and military populations (i.e., Jha \& Kiyonaga, 2010; Jha et al., 2017). Figure 1 presents a schematic of the progression of each trial of the WM task. During each trial, participants were instructed to keep their gaze in the center of the screen at all times. Trials began with a memory array (S1) presented for $3,000 \mathrm{~ms}$, containing either two memory items (high mnemonic load) or one memory item paired with a noise mask (low mnemonic load). S1 was followed by a delay interval of 3,000 ms, which included a task-irrelevant distractor image (neutral or negative in valence) displayed for 2,000 ms.
Following the delay, a single test item (S2) was presented for up to 2,500 ms. Participants were instructed to press a designated key corresponding to whether S2 was an image that had appeared in S1 (match trials) or a novel image (nonmatch trials) that had not appeared in S1. Participants were asked to respond quickly and accurately, with greater emphasis on accuracy. S2 was always of the same category (face or shoe) as S1. The memory item(s) and distractor stimuli were not repeated during the task, with the exception of S2 on match trials. Half of the trials utilized neutral faces as stimuli, and the other half utilized shoes, with both trial types intermixed throughout the task. In addition, half of the trials utilized neutral distractor images, whereas the remaining trials utilized negative distractor images. The task consisted of a 30trial practice block (with accuracy feedback) and three 30-trial experimental blocks ( 90 total experimental trials). The duration of the task was approximately $20 \mathrm{~min}$, with self-timed breaks between blocks.

Distractor images were drawn from the International Affective Picture System (Lang, Bradley, \& Cuthbert, 2008) and were either neutrally valenced (normative valence: $M=$ 5.36, $S D=1.33$; arousal: $M=3.43, S D=2.03$ ) or negatively valenced (normative valence: $M=2.53, S D=1.54$; arousal: $M$ $=5.91, S D=2.23$ ) (Lang et al., 2008). The negative images consisted of aggressive or aversive scenes and objects, whereas the neutral images were matched to the negative images in terms of scene composition and chromatic structure.

Thus, cognitive demands were manipulated along two levels of mnemonic load (low vs. high) and two levels of affective interference (neutral vs. negative distractors), yielding four distinct trial types: low load/neutral distractor, low load/negative distractor, high load/neutral distractor, and high load/negative distractor. Each trial type occurred with approximately equal frequencies (low load/neutral distractor

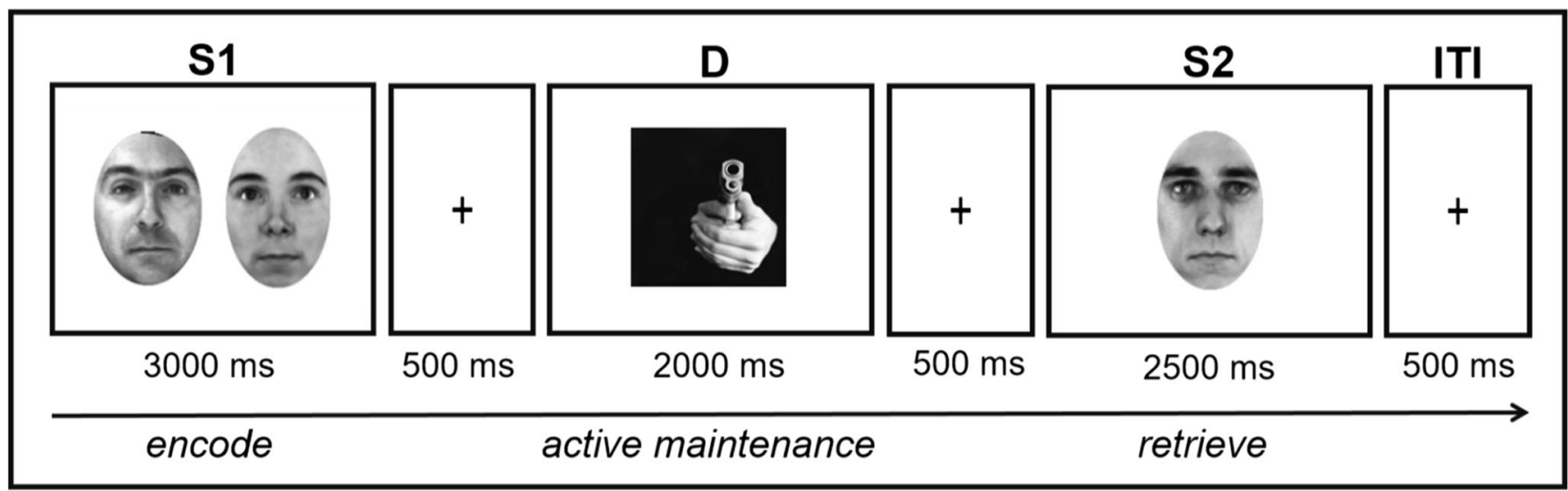

Fig. 1 Time course progression of a sample delayed-recognition working memory task trial (high mnemonic load, negative distractor). Lowmnemonic-load trials utilized a noise mask in place of the second image in S1. During a high-load trial, participants were shown two images of either faces or shoes (S1) and asked to remember them over a delay interval, during which they were shown a distractor image $(\mathrm{D}$, either neutral or negative in valence). After the delay interval, participants were shown a single face or shoe (S2) and asked to determine whether the image matched either of the images seen in S1. The S1 image type (faces vs. shoes) varied randomly across trials, but the S2 type always matched the S1 type within trials. 
condition, 22 trials; low load/negative distractor condition, 23 trials; high load/neutral distractor condition, 23 trials; high load/negative distractor condition, 22 trials). Across the experiment, trials varied across four factors: S1/S2 category (faces/ shoes), match versus nonmatch trials, mnemonic load level (low/high), and affective interference (neutral/negative). The trial order was pseudo-randomly intermixed in terms of these four variables so that identical trial types were never consecutively presented.

Image ratings After completing the WM task, participants were asked to rate the arousal and valence of the images presented during the delay, utilizing a 9-point scale ranging from 1 to 9 . For valence, 1 represented highly negative emotional content, 5 represented neutral emotional content, and 9 represented highly positive emotional content. For arousal, 1 represented the lowest level of arousal and 9 represented the highest level of arousal. Participants were given as much time as needed to complete the rating scales.

Participants rated images in accordance with Lang et al. (2008), which confirmed our manipulation of negative and neutral distractors in the task. The neutral distractors $(M=$ $5.30, S D=0.48)$ were rated as less negative than the negative distractors $(M=2.28, S D=0.68), t(77)=-29.76, p<.001$, $95 \%$ CI $[-3.22,-2.82], d_{z}=3.37$. Additionally, the neutral distractors $(M=2.16, S D=1.45)$ were also rated as less arousing than the negative distractors $(M=3.69, S D=2.12)$, $t(77)=5.26, p<.001,95 \%$ CI $[0.95,2.11], d_{z}=0.596$.

\section{Procedures}

Trained experimenters proctored the administration of the WM task to groups of up to ten participants, each at their own PC laptop workstation. Testing occurred in a quiet room, where participants sat approximately $57 \mathrm{~cm}$ from a PC laptop display and performed the task, image ratings, and questionnaires. At the end of the test battery, participants viewed a decompression condition with positively valenced images to counterbalance any sustained effect of negative mood induced by viewing the negative images. Each session lasted approximately 2 hours and comprised the WM task, as well as other behavioral tasks and questionnaires outside the scope of the present study. All procedures were approved by the Institutional Review Boards of the University of Miami.

\section{Analysis}

The primary outcome measure of interest was WM accuracy for each trial, as this aspect of performance was emphasized in task instructions to participants. The focus on WM accuracy is also in line with previous studies involving delayedrecognition WM tasks and examining accuracy as the primary outcome (Dolcos et al., 2008; Dolcos \& McCarthy, 2006; Jha \& Kiyonaga, 2010; Jha et al., 2017). The RT data (in milliseconds) were also analyzed for completeness. RT outliers were assessed by examining standardized residuals of individual means collapsed across trials for RT. RT trials more than four standard deviations from the mean were considered outliers. There was no significant difference between the results when these outliers were excluded or retained; thus, the full dataset, including outliers, was retained for all analyses.

We analyzed accuracy and RT using hierarchical linear modeling (HLM; Woltman, Feldstain, MacKay, \& Rocchi, 2012). HLM allowed us to model current and previous trial demands simultaneously and to nest trial conditions within each participant. Including both current and previous trial demand types in the same model allowed for the independent assessment of the effects of previous trial demands while controlling for current trial demands. Each trial was included as an observation in the analysis. The fixed effects of mnemonic load (low vs. high), affective interference (neutral vs. negative), and their interaction were entered as level-1 predictors nested within each participant. Mnemonic load $(0=$ low, $1=$ high $)$ and affective interference $(0=$ neutral, 1 = negative $)$ were entered as dummycoded factor variables. To examine the effects of previous trial demand on subsequent performance, variables representing the mnemonic load (low vs. high) and affective interference (neutral vs. negative) of each prior trial were created. Thus, mnemonic load and affective interference were entered as current-trial variables in an initial model (Model 1), and previous-trial variables were added to the initial model while controlling for Model 1 (Model 2).

Trial accuracy was analyzed using hierarchical generalized linear models (HGLM) with PROC GLIMMIX in SAS version 9.4. We utilized a Bernoulli distribution for the binary response variable $(0=$ incorrect, $1=$ correct $)$ and a logit link function, with a random intercept for each subject. The link function transformed the distributions of the predictors to fit the dichotomous response distribution. Parameters were estimated utilizing maximum likelihood estimation based on numerical integration (adaptive quadrature). RT was analyzed utilizing hierarchical linear modeling with PROC MIXED in SAS version 9.4, in which RT was a response variable with a continuous distribution. Restricted estimation maximum likelihood was utilized to estimate a random intercept for each subject and the fixed effects. Type III tests of fixed effects are reported herein, alongside odds, parameter estimates, and $95 \%$ confidence intervals. For follow-up comparisons of significant interactions, the significance $\alpha$ threshold was adjusted for multiple comparisons using the Bonferroni method ( $\alpha=$ $.05 /$ number of comparisons). For accuracy, effect sizes are reported as estimated odds ratios (ORs). ORs are used because of the dichotomous coding of accuracy for each trial (correct 
vs. incorrect). For RT, effect sizes were calculated as standardized mean differences based on the observed means and reported as Cohen's $d_{z}$ (Lakens, 2013).

Nine participants were excluded from the analyses on the basis of self-reported psychological diagnoses (depression and/or anxiety) and/or use of psychotropic medications. These conditions have been shown to influence performance on tasks involving IAPS images (Grimm et al., 2009; Hamilton \& Gotlib, 2008; Shah, Klumpp, Angstadt, Nathan, $\&$ Phan, 2009). Whereas, on average, the participants responded to 86.15 trials $(S D=12.01)$, there were two participants who responded to fewer than $1 / 3$ of the task trials ( 24 and 15 trials out of 90 total trials) and were excluded. Thus, 78 participants were retained for the analyses (46 females, 32 males; $M$ age $=19.37$ years, $S D=1.79$ ).

\section{Results}

\section{Current-trial analyses}

Accuracy The pseudo-intraclass correlation (ICC) was evaluated on the null (intercept-only) model by approximating the variance distribution. The null model examining subjects as random intercepts (pseudo-ICC $=.14$ ) suggested that $14 \%$ of the variability in task accuracy could be explained by between-subjects differences. Thus, the majority $(\sim 86 \%)$ of the variance in WM accuracy occurred within subjects, indicating considerable fluctuations in WM performance over trials. The observed means and standard deviations for accuracy are shown in Table 1.

To examine the impact of current trial demands on accuracy, the first analysis (Model 1) included the fixed effects of mnemonic load (low vs. high), affective interference (neutral vs. negative distractors), and their interaction (see Table 2 for the parameter estimates). We observed a significant main effect of current mnemonic load, $F(1,6939)=106.73, p<.001$; a main effect of current affective interference, $F(1,6939)=$ $35.64, p<.001$; and no significant interaction between load and interference, $F(1,6939)=1.48, p=.224$ (Fig. 2a). Specifically, low-load trials (odds $=16.72$ ) were more than twice as likely to be correct $(\mathrm{OR}=2.40, p<.001,95 \% \mathrm{CI}$
$[2.03,2.83]$ ), when compared to high-load trials (odds $=6.97$ ). In other words, low-load trials had odds $=16.72$, which refers to the odds that any particular low-load trial will be correct versus incorrect. Thus, roughly 16.7 trials were likely to be correct for every one incorrect trial when the trial was low load. High-load trials had odds $=6.97$. Thus, roughly seven high-load trials were likely to be correct for every one incorrect trial. The OR between these effects was equal to $2.40(\sim$ 16.72/6.97), which indicates that the odds of a low-load trial being correct were more than twice as likely as the odds of a high-load trial being correct. For affective interference, neutral-distractor trials (odds $=13.89$ ) were almost twice as likely to be correct $(\mathrm{OR}=1.66, p<.001,95 \% \mathrm{CI}[1.40,1.95])$, when compared to negative-distractor trials (odds $=8.39$ ).

RT The null model examining within- and between-subjects differences demonstrated that ICC $=.19$, suggesting that $19 \%$ of the variance in RTs could be explained by between-subjects differences. Similar to the results for accuracy, $81 \%$ of the variance in RT could be attributed to within-subjects differences. The observed means and standard deviations for RTs are shown in Table 1.

To examine the impact of current trial demands on RTs, the first analysis (Model 1) included fixed effects of mnemonic load (low vs. high), affective interference (neutral vs. negative distractors), and their interaction (see Table 3 for the parameter estimates). The RT results mirrored the accuracy results. We observed a significant main effect of current mnemonic load, $F(1,6939)=369.74, p<.001$; a main effect of current affective interference, $F(1,6939)=45.86, p<.001$; and no significant interaction between load and affective interference, $F(1$, $6939)=1.96, p=.162$ (Fig. 3a). Specifically, low-load trials were significantly faster than high-load trials $(\beta=-139.30, p$ $\left.<.001, d_{z}=0.78\right)$, and neutral-distractor trials were significantly faster than negative-distractor trials $(\beta=-49.06, p<$ $\left..001, d_{z}=0.29\right)$.

\section{Previous-trial analyses}

Accuracy To examine the impact of the previous trial demands on subsequent trial performance, we included the fixed effects

Table 1 Observed means and standard deviations for Experiments 1 and 2

\begin{tabular}{|c|c|c|c|c|c|c|c|c|}
\hline & \multicolumn{4}{|l|}{ Current Trial } & \multicolumn{4}{|l|}{ Previous Trial } \\
\hline & Low-Neutral & Low-Negative & High- Neutral & High- Negative & Low-Neutral & Low-Negative & High-Neutral & High-Negative \\
\hline \multicolumn{9}{|l|}{ Experiment 1} \\
\hline Accuracy & $94.11 \%(8.36)$ & $91.58 \%(8.76)$ & $88.46 \%$ (9.32) & $81.35 \%(12.09)$ & $84.17 \%(10.79)$ & $89.46 \%$ (8.82) & $89.86 \%$ (8.77) & $91.33 \%$ (9.94) \\
\hline RT & 713.20 (149.98) & $752.12(156.11)$ & $842.36(170.36)$ & 901.56 (196.97) & 829.21 (171.66) & 793.35 (162.47) & $797.83(159.34)$ & 795.49 (172.32) \\
\hline \multicolumn{9}{|c|}{ 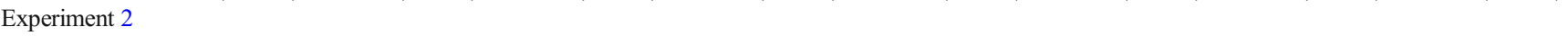 } \\
\hline Accuracy & $95.93 \%(6.41)$ & $92.79 \%(5.70)$ & $90.10 \%(9.22)$ & $81.28 \%(9.73)$ & $85.92 \%(8.51)$ & $91.13 \%(7.53)$ & $89.93 \%$ (8.19) & $92.92 \%(7.11)$ \\
\hline RT & 741.46 (145.28) & 807.70 (161.67) & $888.28(150.47)$ & $964.54(177.80)$ & 896.25 (176.85) & $842.76(160.67)$ & $849.72(145.68)$ & $821.44(152.01)$ \\
\hline
\end{tabular}

Observed means and standard deviations (in parentheses) are provided for accuracy and response time (RT) outcome variables. 
Table 2 Experiment 1 task accuracy

\begin{tabular}{|c|c|c|}
\hline \multirow[t]{2}{*}{ Model Effects } & \multicolumn{2}{|l|}{ Log-Odds $(S E)$} \\
\hline & Model 1 & Model 2 \\
\hline \multicolumn{3}{|l|}{ Fixed Effects } \\
\hline Intercept & $3.017(0.138)^{* *}$ & $2.552(0.149)^{* *}$ \\
\hline Current Load & $-.772(.129)^{* *}$ & $-.757(.133)^{* *}$ \\
\hline Current Interference & $-.402(.136)^{* *}$ & $-.393(.137)^{* *}$ \\
\hline Previous Load & - & $.586(.110)^{* *}$ \\
\hline Previous Interference & - & $.514(.109)^{* *}$ \\
\hline Current Load $\times$ Current Interference & $-.206(.169)$ & $-.216(.172)$ \\
\hline Previous Load $\times$ Previous Interference & - & $-.365(.164)^{*}$ \\
\hline \multicolumn{3}{|l|}{ Random Effects } \\
\hline Intercept Variance & 0.561 & 0.547 \\
\hline$N_{\text {obs }}$ & 7,020 & 6,786 \\
\hline \multicolumn{3}{|l|}{ Fit Statistics } \\
\hline -2 Log-Likelihood & $4,508.12$ & $4,351.39$ \\
\hline
\end{tabular}

Maximum likelihood estimates are reported as log-odds, and standard errors are reported in parentheses. Low-load/neutral-distractor trials served as the reference condition. $N_{\mathrm{obs}}=$ number of observations. Significant effects are indicated by asterisks: ${ }^{*} p<.05,{ }^{* *} p<.01$.

of previous mnemonic load and previous-trial affective interference, and the interaction between previous load and previous affective interference, in a second model (Model 2) alongside the fixed effects from Model 1. Consistent with Model 1, we observed main effects of current load and affective interference. In addition, we observed a significant main effect of previous mnemonic load, $F(1,6702)=24.26, p<.001$; a main effect of previous affective interference, $F(1,6702)=16.39, p$ $<.001$; and a significant interaction between previous load and previous interference, $F(1,6702)=4.99, p=.026$ (Table 2). For mnemonic load, trials preceded by high-load trials (odds $=13.24$ ) were more likely to be correct $(\mathrm{OR}=$ $1.50, p<.001,95 \%$ CI $[1.28,1.76])$ than trials preceded by low-load trials (odds $=8.85$ ). For affective interference, trials preceded by negative-distractor trials (odds $=12.78$ ) were more likely to be correct $(\mathrm{OR}=1.39, p<.001,95 \% \mathrm{CI}$ $[1.19,1.64])$ than trials preceded by neutral-distractor trials (odds = 9.18) (Fig. 2b).

The significant interaction between previous mnemonic load and previous affective interference was further examined through follow-up comparisons. Overall, these comparisons revealed that accuracy was higher on the three previous-trial conditions with high demands (high load/ negative distractors, high load/neutral distractors, and low load/negative distractors) than in the lowest-demand condition (low load/neutral distractors). Specifically, trials preceded by high-load/negative-distractor trials (odds = 14.27) were twice as likely to be correct $(\mathrm{OR}=2.08, p$ $<.001,95 \%$ CI $[1.66,2.62])$ as trials preceded by lowload/neutral-distractor trials (odds $=6.85$ ). Similarly, trials preceded by high-load/neutral-distractor trials (odds =
12.30) or low-load/negative-distractor trials (odds = 11.44) were almost twice as likely be correct $(\mathrm{OR}=$ $1.80, p<.001,95 \%$ CI $[1.45,2.23]$, and OR $=1.67, p$ $<.001,95 \%$ CI $[1.35,2.07]$, respectively) as low-load/ neutral-distractor trials (Fig. 2c). These three comparisons survived Bonferroni correction (.05/6 comparisons, $p \mathrm{~s}<$ $.008)$.

However, follow-up comparisons did not reveal significant differences between the three previous-trial conditions with high demands (high load/negative distractors, high load/neutral distractors, and low load/negative distractors). Specifically, trials preceded by high-load/neutral-distractor trials or low-load/negative-distractor trials were no more likely to be correct $(\mathrm{OR}=1.16, p=.224$, $95 \%$ CI $[0.91,1.47]$ and $\mathrm{OR}=1.25, p=.069,95 \% \mathrm{CI}$ $[0.98,1.58]$, respectively) than were trials preceded by high-load/negative-distractor trials. Furthermore, trials preceded by high-load/neutral-distractor trials were no more likely to be correct $(\mathrm{OR}=1.08, p=.529,95 \% \mathrm{CI}$ $[0.86,1.35])$ than trials preceded by low-load/negativedistractor trials. Taken together, these results suggest that the benefits to accuracy from preceding high-load and negative-distractor trials were no greater than the effect of either demand type alone.

RT To examine the impact of previous trial demands on the subsequent trial RT, we included the fixed effects of previous mnemonic load, previous affective interference, and the interaction between previous load and affective interference, in a second model (Model 2), alongside the fixed effects from Model 1 (Table 3). The RT results were consistent with the 
a

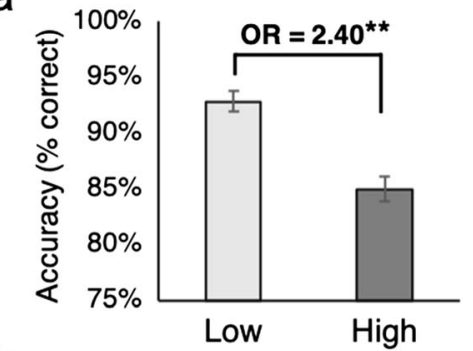

b
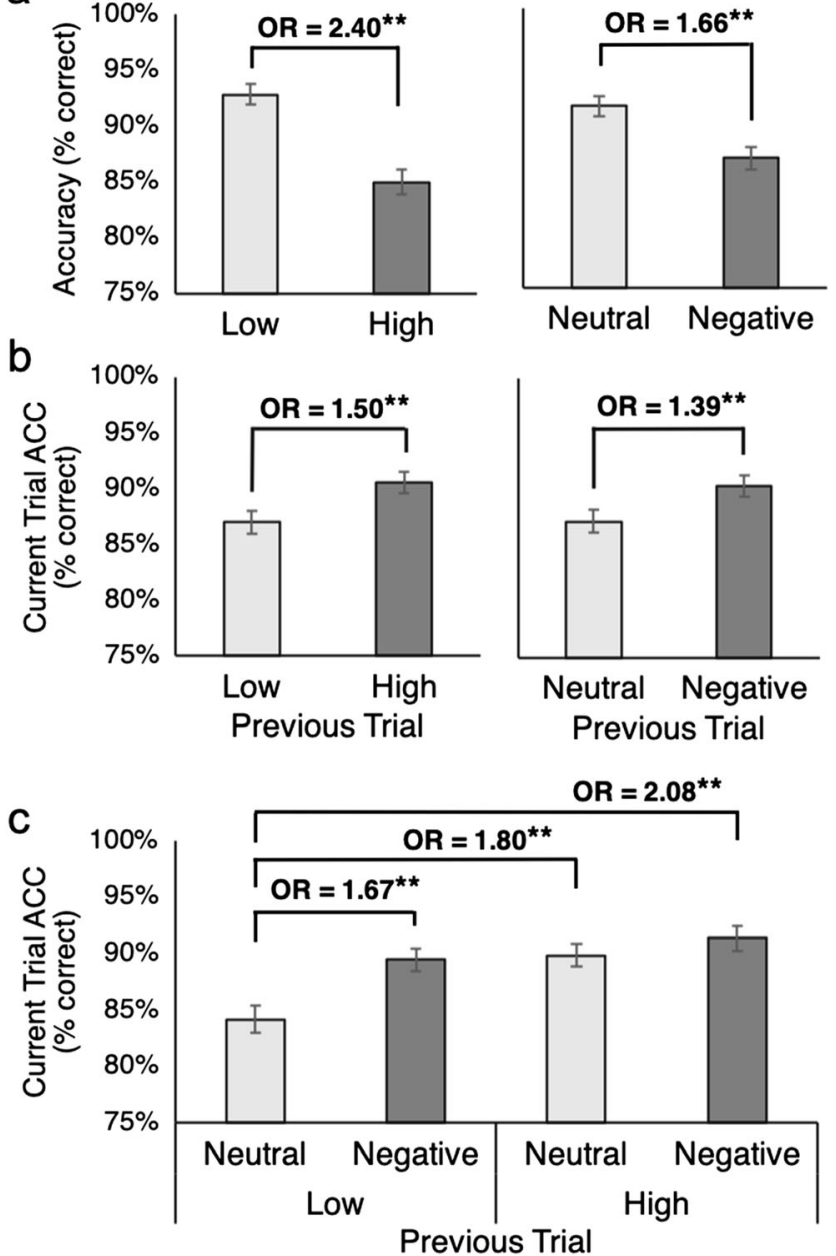

Fig. 2 Effects of current and previous trial demands on accuracy for Experiment 1. The observed data are plotted as mean percentages correct for all trials, collapsed across individuals. (a) Current-trial main effects of mnemonic load (low, high) and affective interference (neutral, negative). (b) Previous-trial main effects of mnemonic load (low, high) and affective interference (neutral, negative). (c) Previous-trial demand effects on current-trial accuracy. Hierarchical linear modeling analyses yielded odds ratios (ORs), which are indicated with asterisks where significant. ${ }^{* *} p<.01$

accuracy results. Consistent with Model 1, we observed main effects of current load and affective interference. In addition, we observed a significant main effect of previous mnemonic load, $F(1,6702)=4.88, p=.027$; a main effect of previous affective interference, $F(1,6702)=4.05, p=.044$; and a significant interaction between previous load and previous affective interference, $F(1,6702)=6.58, p=.010$. Specifically, trials preceded by high-load trials were significantly faster than trials preceded by low-load trials $(\beta=-$ $16.38, p=.027, d_{z}=0.09$ ), and trials preceded by negativedistractor trials were significantly faster than those preceded by neutral-distractor trials $\left(\beta=-14.92, p=.044, d_{z}=0.14\right)$ (Fig. 3b).

The significant interaction revealed that RTs were faster on all three previous-trial conditions with high demands (high load/negative distractors, high load/neutral distractors, and low load/negative distractors) than on the lowest-demand condition (low load/neutral distractors), and RTs did not differ significantly between the three previous-trial conditions with high demands. Specifically, trials preceded by high-load/negative-distractor trials $\left(\beta=-31.30, p=.004, d_{z}=0.19\right)$, highload/neutral-distractor trials $\left(\beta=-35.38, p<.001, d_{z}=0.16\right)$, and low-load/negative-distractor trials $(\beta=-33.93, p=.001$, $\left.d_{z}=0.20\right)$ all had significantly faster RTs than trials preceded by low-load/neutral-distractor trials. These three comparisons survived Bonferroni correction (six comparisons, $p \mathrm{~s}<.008$ ).

However, trials preceded by high-load/negative-distractor trials did not demonstrate significantly different RTs than trials preceded by high-load/neutral-distractor trials $(\beta=4.08, p=$ .695 ) or trials preceded by low-load/negative-distractor trials $(\beta=2.63, p=.801)$. Furthermore, trials preceded by highload/neutral-distractor trials did not demonstrate significant different RTs than trials preceded by low-load/negativedistractor trials $(\beta=-1.45, p=.801)$ (Fig. 3c).

\section{Discussion}

Consistent with previously reported findings, higher (vs. lower) levels of current-trial task demands reduced current-trial performance (Dolcos et al., 2008; Dolcos \& McCarthy, 2006; Jha et al., 2017) but enhanced performance on subsequent trials. Specifically, high mnemonic load (as compared to low mnemonic load) and negative distractors (as compared to neutral distractors) resulted in enhanced performance on the subsequent trial. A significant interaction between previous mnemonic load and previous affective interference was observed. Trials preceded by trials with both low load and neutral distractors had lower performance than those preceded by trials with high load and/or negative distractors. Overall, our results demonstrate that both cognitive and affective demands can upregulate cognitive control resources to benefit subsequent performance in a WM task.

\section{Experiment 2}

The results of Experiment 1 replicated the results of Jha and Kiyonaga (2010) regarding performance benefits as a function of previous trials with high versus low mnemonic load. In addition, as per the predictions of prior studies in which negative affect had been found to upregulate cognitive control (e.g., Dreisbach \& Fischer, 2012a), performance benefits of previous trials with negative versus neutral interference were also observed. To support the validity of these findings, a replication study preregistered within the Open Science Framework (https:// osf.io/v8nm5/) was conducted. 
Table 3 Experiment 1 task response time (RT)

\begin{tabular}{|c|c|c|}
\hline \multirow[t]{2}{*}{ Model Effects } & \multicolumn{2}{|c|}{ Parameter Estimates $(S E)$} \\
\hline & Model 1 & Model 2 \\
\hline \multicolumn{3}{|l|}{ Fixed Effects } \\
\hline Intercept & $713.20(18.84)^{* *}$ & $737.21(20.12)^{* *}$ \\
\hline Current Load & $129.16(10.25)^{* *}$ & $136.48(10.66)^{* *}$ \\
\hline Current Interference & $38.92(10.25)^{* *}$ & $41.16(10.42)^{* *}$ \\
\hline Previous Load & - & $-35.38(10.55)^{* *}$ \\
\hline Previous Interference & - & $-33.93(10.55)^{* *}$ \\
\hline Current Load $\times$ Current Interference & $20.28(14.49)$ & $12.73(14.81)$ \\
\hline Previous Load $\times$ Previous Interference & - & $38.01(14.82)^{*}$ \\
\hline \multicolumn{3}{|l|}{ Random Effects } \\
\hline Intercept Variance & 23,492 & 23,656 \\
\hline Residual Variance & 92,060 & 92,802 \\
\hline$N_{\text {obs }}$ & 7,020 & 6,786 \\
\hline \multicolumn{3}{|l|}{ Fit Statistics } \\
\hline-2 Log-Likelihood & $100,379.3$ & $97,070.3$ \\
\hline
\end{tabular}

Maximum likelihood estimates are reported, and standard errors are reported in parentheses. Low-load/neutral-distractor trials served as the reference condition. $N_{\mathrm{obs}}=$ number of observations. Significant effects are indicated by asterisks: ${ }^{*} p<.05,{ }^{* *} p<.01$.

\section{Method}

\section{Participants}

One-hundred healthy young adults were recruited from the University of Miami community (79 females, 21 males; $M$ age $=20.25$ years, $S D=1.72$ ), who received course credit or monetary compensation for their participation. Informed consent was obtained in accordance with the Institutional Review Boards of the University of Miami.

\section{Experimental stimuli and design}

WM task with affective distractors Participants completed the same WM task that we described in Experiment 1 (see Fig. 1 for a schematic of the progression of each trial of the WM task).

Image rating After completing the WM task, participants were asked to rate the arousal and valence of the images as described in Experiment 1. Neutral distractors $(M=5.38$, $S D=0.59)$ were rated as being less negative than negative distractors $(M=2.21, S D=0.62), t(75)=-34.42, p<$ $.001,95 \%$ CI $[-3.35,-2.98], d_{z}=3.95$. Additionally, neutral distractors $(M=2.20, S D=1.34)$ were also rated as being less arousing than negative distractors $(M=4.11$, $S D=2.07), t(75)=7.50, p<.001,95 \%$ CI $[1.41,2.42], d_{z}$ $=0.861$.

\section{Procedures}

All procedures were identical to those described in Experiment 1, with the exception that the study session lasted approximately 1 hour and comprised only the WM task, along with questionnaires outside the scope of the present study. All procedures were approved by the Institutional Review Boards of the University of Miami.

\section{Analysis}

Analyses methods replicated those described in Experiment 1. The primary outcome measure of interest was accuracy for each trial (i.e., correct vs. incorrect), but response time data (RT, in milliseconds) were also analyzed for completeness. RT outliers were assessed by examining standardized residuals of individual means collapsed across trials for RT, and RT trials more than four standard deviations from the mean were considered outliers. No trials met the exclusion criteria and, thus, the full dataset was retained for all analyses. Twenty-four participants were excluded from analyses on the basis of selfreported psychological diagnoses (depression and/or anxiety) and/or use of psychotropic medications. All remaining participants responded to more than $1 / 3$ of the task trials and thus were retained for the analyses. After exclusions, the data from 76 participants were analyzed ( 60 females, 16 males; $M$ age $=$ 20.29 years, $S D=1.90$ ).

We analyzed the dependent measures using HLM (Woltman et al., 2012), in which each trial was included as 


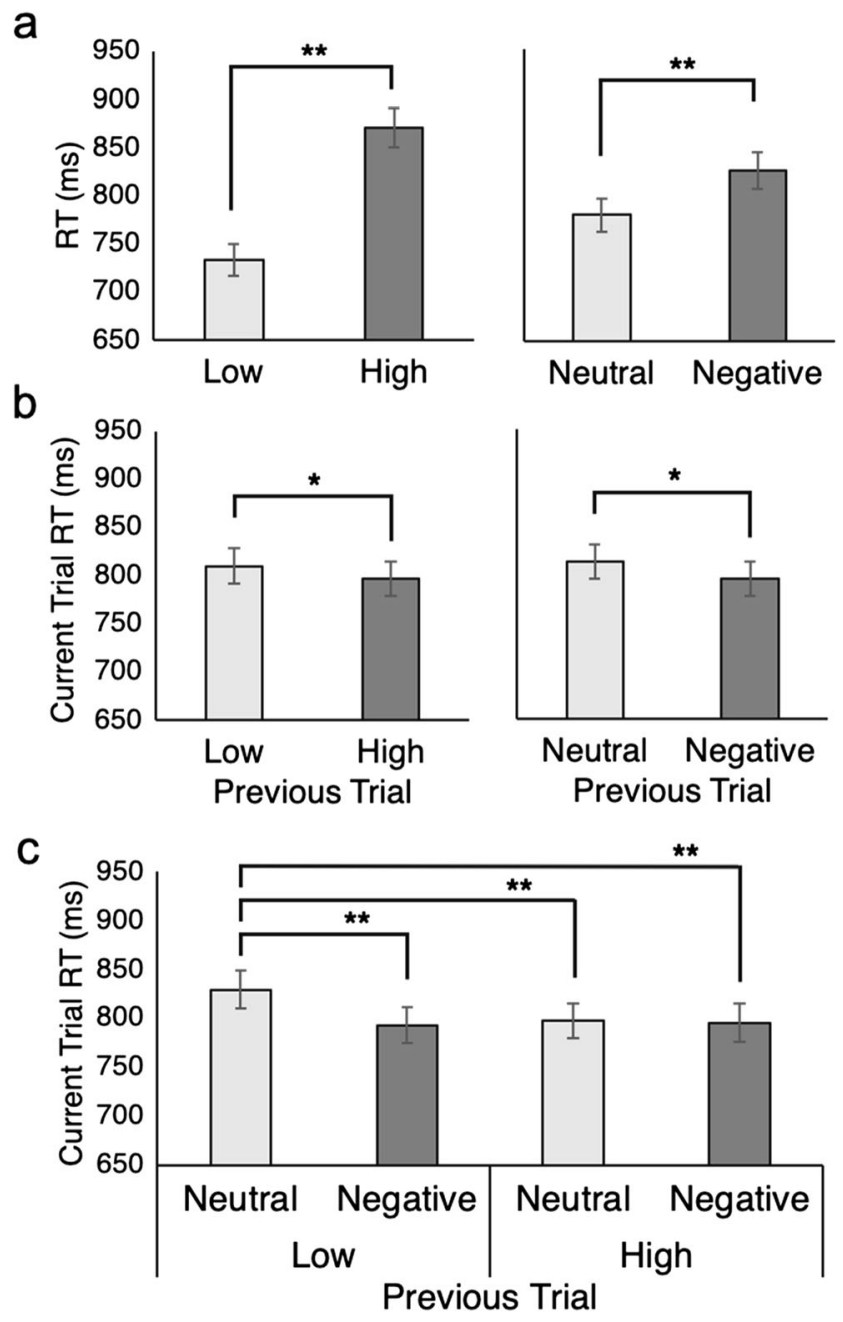

Fig. 3 Effects of current and previous trial demands on response time (RT) for Experiment 1. The observed data are plotted as mean RTs for all trials, collapsed across individuals. (a) Current-trial main effects of mnemonic load (low, high) and affective interference (neutral, negative). (b) Previous-trial main effects of mnemonic load (low, high) and affective interference (neutral, negative). (c) Previous-trial demand effects on current-trial RTs. ${ }^{*} p<.05,{ }^{* * *} p<.01$

an observation in the analysis. We analyzed fixed effects of current mnemonic load (low vs. high), current affective interference (neutral vs. negative), the interaction between current mnemonic load and current affective interference, previous mnemonic load (low vs. high), previous affective interference (neutral vs. negative), and the interaction between previous mnemonic load and previous affective interference as level-1 predictors nested within each participant.

\section{Results}

\section{Accuracy}

The null model examining subjects as random intercepts (pseudo-ICC $=.08$ ) suggested that $8 \%$ of the variability in task accuracy could be explained by between-subjects differences. Thus, the preponderance $(\sim 92 \%)$ of the variance in WM accuracy occurred within subjects. The observed means and standard deviations for accuracy are shown in Table 1.

To examine the impact of current and previous trial demands on accuracy, we included the fixed effects of current mnemonic load, current affective interference, and their interaction, as well as previous mnemonic load, previous affective interference, and their interaction. We observed a significant main effect of current mnemonic load, $F(1,6530)=111.33, p$ $<.001$; a main effect of current affective interference, $F(1$, $6530)=56.02, p<.001$; and no significant interaction between load and affective interference, $F(1,6530)=1.40, p=$ .236 (see Table 4 for the parameter estimates). Specifically, low-load trials (odds $=20.17$ ) were more than twice as likely to be correct $(\mathrm{OR}=2.75, p<.001,95 \% \mathrm{CI}[2.28,3.31])$ as high-load trials (odds $=7.35$ ). Furthermore, neutral-distractor trials (odds $=17.42)$ were twice as likely to be correct $(\mathrm{OR}=$ $2.05, p<.001,95 \%$ CI $[1.70,2.47])$ as negative-distractor trials (odds $=8.51)$ (Fig. 4a).

We observed a significant main effect of previous mnemonic load, $F(1,6530)=15.37, p<.001$; a main effect of previous affective interference, $F(1,6530)=28.42, p<.001$; and no significant interaction between previous load and previous affective interference, $F(1,6530)=1.33, p=.249$. Specifically, trials preceded by high-load trials (odds $=$ 14.44) were more likely to be correct ( $\mathrm{OR}=1.41, p<.001$, $95 \%$ CI $[1.19,1.67])$ than trials preceded by low-load trials (odds $=10.26$ ). In addition, trials preceded by negativedistractor trials (odds $=15.36$ ) were more likely to be correct $(\mathrm{OR}=1.59, p<.001,95 \% \mathrm{CI}[1.34,1.89])$ than trials preceded by neutral-distractor trials (odds $=9.65$ ) (Fig. 4b).

Although the interaction between previous load and affective interference was not statistically significant, we observed similar patterns of means and ORs for the previous-trial conditions in both experiments (see Table 1). Thus, we conducted the same follow-up comparisons reported in Experiment 1 to better understand which previous-trial conditions were driving the presence or absence of significant condition differences.

Similar to Experiment 1, the results demonstrated that the current-trial accuracy was higher on all three previous-trial conditions with high demands (high load/negative distractors, high load/neutral distractors, and low load/negative distractors) than in the lowest-demand condition (low load/ neutral distractors). Specifically, trials preceded by highload/negative-distractor trials (odds $=17.33$ ) were more than twice as likely to be correct ( $\mathrm{OR}=2.24, p<.001,95 \% \mathrm{CI}$ $[1.75,2.87])$ as trials preceded by low-load/neutral-distractor trials (odds $=7.74$ ). In addition, trials preceded by high-load/ neutral-distractor trials (odds $=12.04$ ) or low-load/negativedistractor trials (odds $=13.61$ ) were more likely to be correct $(\mathrm{OR}=1.56, p<.001,95 \% \mathrm{CI}[1.25,1.94]$, and $\mathrm{OR}=1.76, p<$ $.001,95 \% \mathrm{CI}[1.40,2.21]$, respectively) than trials preceded 
Table 4 Experiment 2 task accuracy (ACC) and response time (RT)

\begin{tabular}{|c|c|c|}
\hline Model Effects & $\begin{array}{l}\text { ACC } \\
\text { Log-Odds }(S E)\end{array}$ & $\begin{array}{l}\text { RT } \\
\text { Parameter Estimates }(S E)\end{array}$ \\
\hline \multicolumn{3}{|l|}{ Fixed Effects } \\
\hline Intercept & $2.852(0.156)^{* * *}$ & $787.49(19.47)^{* *}$ \\
\hline Current Load & $-.897(.153)^{* *}$ & $147.25(10.45)^{* *}$ \\
\hline Current Interference & $-.603(.157)^{* *}$ & $66.09(10.22)^{* *}$ \\
\hline Previous Load & $.442(.113)^{* *}$ & $-51.26(10.35)^{* *}$ \\
\hline Previous Interference & $.565(.116)^{* *}$ & $-51.82(10.35)^{* *}$ \\
\hline Current Load $\times$ Current Interference & $-.226(.191)$ & $8.36(14.53)$ \\
\hline Previous Load $\times$ Previous Interference & $-.201(.174)$ & $30.30(14.54)^{*}$ \\
\hline \multicolumn{3}{|l|}{ Random Effects } \\
\hline Intercept Variance & 0.316 & 21,367 \\
\hline Residual Variance & - & 87,040 \\
\hline$N_{\text {obs }}$ & 6,612 & 6,612 \\
\hline \multicolumn{3}{|l|}{ Fit Statistics } \\
\hline-2 Log-Likelihood & $3,945.42$ & $94,153.8$ \\
\hline
\end{tabular}

For accuracy, maximum likelihood estimates are reported as log-odds, and standard errors are reported in parentheses. For RTs, maximum likelihood estimates are reported, and standard errors are reported in parentheses. Low-load/neutral-distractor trials served as the reference condition. $N_{\text {obs }}=$ number of observations. Significant effects are indicated by asterisks: ${ }^{*} p<.05,{ }^{* *} p<.01$.

by low-load/neutral-distractor trials. These three comparisons survived Bonferroni correction (six comparisons, $p$ s $<.008$ ).

Also similar to Experiment 1, trials preceded by high-load/ negative-distractor trials were no more likely to be correct $(\mathrm{OR}=1.27, p=.069,95 \% \mathrm{CI}[0.98,1.65])$ than trials preceded by low-load/negative-distractor trials. Furthermore, trials preceded by high-load/neutral-distractor trials were no more likely to be correct $(\mathrm{OR}=0.884, p=.304,95 \%$ CI $[0.70$, 1.12]) than trials preceded by low-load/negative-distractor trials. However, unlike in Experiment 1, trials preceded by highload/negative-distractor trials were significantly more likely to be correct $(\mathrm{OR}=1.44, p=.005,95 \%$ CI $[1.12,1.86])$ than trials preceded by high-load/neutral-distractor trials, and this comparison survived Bonferroni correction. Overall, the results of both experiments suggest that trials were more likely to be correct when they were preceded by high-demand trials than when preceded by low-load/neutral-distractor trials (see Fig. 4c).

\section{RT}

The null model examining within- and between-subjects differences demonstrated an ICC $=.18$, suggesting that $18 \%$ of the variance in RT could be explained by between-subjects differences. Thus, the majority (82\%) of the variance in RTs could be attributed to within-subjects differences. The observed means and standard deviations for RTs are shown in Table 1.

To examine the impact of current and previous trial demands on RTs, we included the fixed effects of current mnemonic load, current affective interference, and their interaction, as well as previous mnemonic load, previous affective interference, and their interaction. We observed a significant main effect of current mnemonic load, $F(1,6530)=434.25 p$ $<.001$; a main effect of current affective interference, $F(1$, $6530)=93.60, p<.001$; and no significant interaction between load and affective interference, $F(1,6530)=0.33, p=$ .565 (see Table 4 for the parameter estimates). Specifically, low-load trials were significantly faster than high-load trials ( $\beta$ $\left.=-151.43, p<.001, d_{z}=0.96\right)$, and neutral-distractor trials were significantly faster than negative-distractor trials $(\beta=$ 70.26, $p<.001, d_{z}=0.41$ ) (Fig. 5a).

We also observed a significant main effect of previous-trial mnemonic load, $F(1,6530)=24.64, p=.027$; a main effect of previous affective interference, $F(1,6530)=25.41, p=.044$; and a significant interaction between previous load and previous affective interference, $F(1,6530)=4.34, p=.037$. Specifically, trials preceded by high-load trials were significantly faster than trials preceded by low-load trials $(\beta=-$ $36.11, p<.001, d_{z}=0.21$ ), and trials preceded by negativedistractor trials were significantly faster than those preceded by neutral-distractor trials $\left(\beta=-36.67, p<.001, d_{z}=0.29\right)$ (Fig. 5b).

Similar to Experiment 1, the significant interaction revealed that RTs were faster in all three previous-trial conditions with high demands (high load/negative distractors, high load/neutral distractors, and low load/negative distractors) than in the lowest-demand condition (low load/neutral distractors). Specifically, trials preceded by high-load/negative-distractor trials $(\beta=-72.78, p<$ 

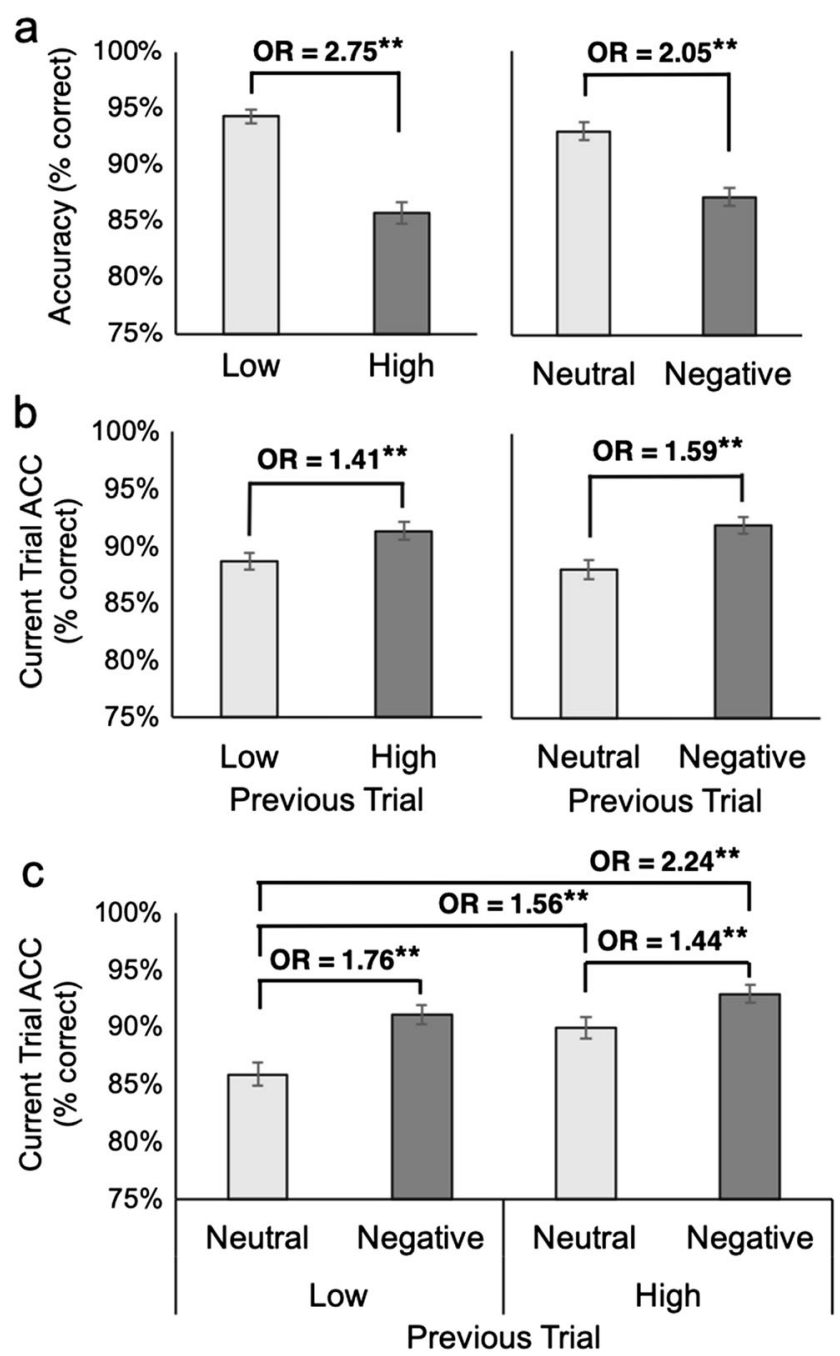

Fig. 4 Effects of current and previous trial demands on accuracy for Experiment 2. The observed data are plotted as mean percentages correct for all trials, collapsed across individuals. (a) Current-trial main effects of mnemonic load (low, high) and affective interference (neutral, negative). (b) Previous-trial main effects of mnemonic load (low, high) and affective interference (neutral, negative). (c) Previous-trial demands (low load/neutral distractor, low load/negative distractor, high load/ neutral distractor, high load/negative distractor) on current-trial accuracy. Hierarchical linear modeling analyses yielded odds ratios (ORs), which are indicated with asterisks where significant. ${ }^{* *} p<.01$

$\left..001, d_{z}=0.38\right)$, high-load/neutral-distractor trials $(\beta=$ $\left.51.26, p<.001, d_{z}=0.26\right)$, and low-load/negativedistractor trials $\left(\beta=51.82, p<.001, d_{z}=0.28\right)$ had significantly faster RTs than trials preceded by low-load/neutral-distractor trials, and these three comparisons survived Bonferroni correction (six comparisons, $p \mathrm{~s}<.008$ ).

Trials preceded by high-load/negative-distractor trials did not demonstrate significantly different RTs than trials preceded by high-load/neutral-distractor trials $(\beta=-21.52, p=.035$, $\left.d_{z}=0.18\right)$ or trials preceded by low-load/negative-distractor trials $\left(\beta=-20.96, p=.040, d_{z}=0.11\right)$, as these comparisons did not survive Bonferroni correction (six comparisons, $p \mathrm{~s}<$ .008 ). Furthermore, trials preceded by high-load/neutral- a
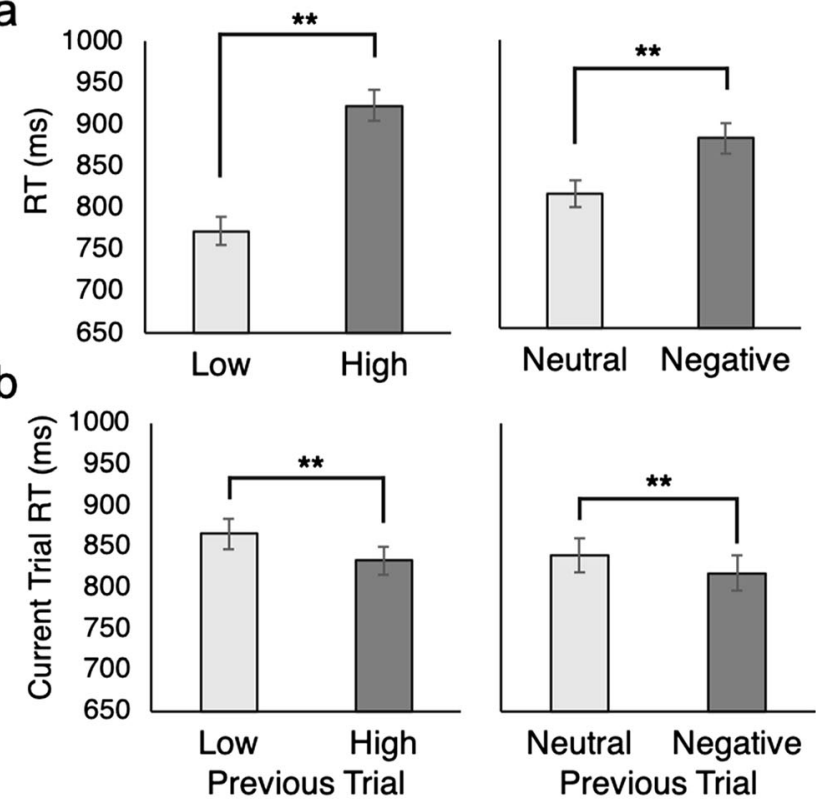

C

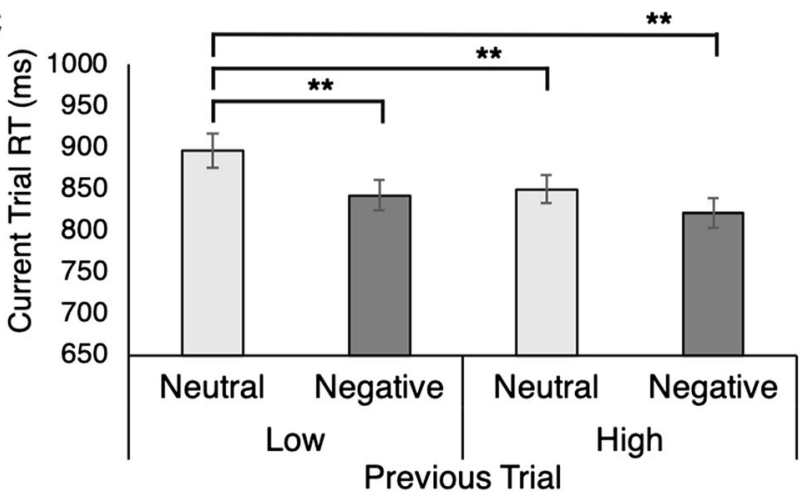

Fig. 5 Effects of current and previous trial demands on response time (RT) for Experiment 2. The observed data are plotted as mean RTs for all trials, collapsed across individuals. (a) Current-trial main effects of mnemonic load (low, high) and affective interference (neutral, negative). (b) Previous-trial main effects of mnemonic load (low, high) and affective interference (neutral, negative). (c) Previous-trial demands (low load/ neutral distractor, low load/negative distractor, high load/neutral distractor, high load/negative distractor) on current-trial RTs. ${ }^{* *} p<.01$

distractor trials did not demonstrate significantly different RTs than trials preceded by low-load/negative-distractor trials $(\beta=0.56, p=.956)$ (Fig. $5 c)$.

\section{Combined analyses across Experiments 1 and 2}

We formally examined whether current-trial and previous-trial effects were different between the experiments by adding a fixed factor of experiment (Exp. 1, Exp. 2) to the HLM model. To do so, we combined the data from both experiments and added the interactions between experiment, current load, and current affective interference, as well as the interactions between experiment, previous load, and previous affective interference, to our model. 
Accuracy The results of the combined dataset demonstrated the expected main effects of current load, $F(1,13232)=$ $212.11, p<.001$, and current affective interference, $F(1$, $13232)=89.65, p<.001$, as well as the nonsignificant interaction between current load and affective interference, $F(1$, $13232)=2.98, p=.085$. However, we did not observe any significant interactions between experiment and current load, $F(1,13232)=1.42, p=.234$; current affective interference, $F(1,13232)=2.94, p=.086$; or current load by current affective interference, $F(1,13232)=0.01, p=.954$.

We also observed the expected main effects of previous load, $F(1,13232)=38.79, p<.001$, and previous affective interference, $F(1,13232)=44.39, p<.001$. In addition, we observed a significant interaction between previous load and previous affective interference, $F(1,13232)=5.59, p=.018$. Yet, we did not observe any significant interactions between experiment and previous load, $F(1,13232)=0.24, p=.628$; previous affective interference, $F(1,13232)=1.33, p=.250$; or previous load by previous affective interference, $F(1$, $13232)=0.46, p=.499$.

RT The results of the combined dataset demonstrated the expected main effects of current load, $F(1,13232)=803.64, p<$ .001 , and current affective interference, $F(1,13232)=128.90$, $p<.001$, as well as the nonsignificant interaction between current load and affective interference, $F(1,13232)=1.03, p$ $=.310$. We did not observe any significant interactions between experiment and current load, $F(1,13232)=0.68, p=$ .408 , or current load by current affective interference, $F(1$, $13232)=0.04, p=.833$, but we did find a significant interaction between experiment and current affective interference, $F(1,13232)=4.80, p=.028$. However, RTs on neutraldistractor trials did not differ between experiments $(\beta=$ $36.86, p=.145$ ), and RTs on negative-distractor trials also did not differ between experiments $(\beta=59.60, p=.018)$ after correcting for multiple comparisons (four comparisons, $\alpha=$ $.013)$.

We also observed the expected main effects of previous load, $F(1,13232)=25.51, p<.001$, and previous affective interference, $F(1,13232)=24.65, p<.001$, as well as the interaction between previous load and previous affective interference, $F(1,13232)=10.81, p=.001$. We did not observe any significant interactions between experiment and previous load, $F(1,13232)=3.61, p=.058$, or previous load by previous affective interference, $F(1,13232)=$ $0.14, p=.711$, but we did find a significant interaction between experiment and previous affective interference, $F(1,13232)=4.38, p=.036$. However, RTs on negativedistractor trials did not differ between experiments $(\beta=$ 37.36, $p=.139)$, and RTs on neutral-distractor trials also did not differ between experiments $(\beta=59.10, p=.020)$ after correcting for multiple comparisons (four comparisons, $\alpha=.013$ ).

\section{Discussion}

The results of the replication (Exp. 2) are largely consistent with Experiment 1. As expected, high mnemonic load (vs. low mnemonic load), and negative-distractor trials (vs. neutral distractors) resulted in lower current-trial WM performance. Moreover, high mnemonic load (vs. low mnemonic load), and negative-distractor trials (vs. neutral distractors) resulted in enhanced performance on the subsequent trial. In Experiment 2, we did not observe a significant interaction between previous mnemonic load and previous affective interference for accuracy, but this interaction was significant for RTs. Follow-up analyses of accuracy and RT demonstrated that the differences between the effects of current and previous trial demands were comparable between experiments.

\section{General Discussion}

Across two experiments we investigated if dynamic adjustments in cognitive control were triggered by the manipulation of task demands related to mnemonic load and affective interference within a delayed-recognition WM task. The findings revealed that greater task demands enhanced performance on subsequent trials. Overall, these results demonstrated a replication of a prior study regarding the upregulation of cognitive control as a function of mnemonic demands (Jha \& Kiyonaga, 2010), and extended findings over two experiments to include upregulation as a function of affective interference during WM task performance.

It has been widely established that as greater demands are placed on WM, current-trial performance is reduced. Yet, evidence of the performance costs associated with greater mnemonic load and affective interference stem mainly from separate lines of research. WM performance has been shown to vary inversely with mnemonic load, such that performance decreases as load increases (Jha \& Kiyonaga, 2010; Jha \& McCarthy, 2000). Studies involving affective distraction in delayed-recognition WM tasks have also demonstrated that negative distraction leads to reduced task accuracy (Dolcos $\&$ McCarthy, 2006). In the present experiments, we manipulated both mnemonic load and affective distraction within the same task, demonstrating the performance impairments in trials with both high load and negative distractors. These findings are consistent with a previous study utilizing the same task in a military cohort (Jha et al., 2017).

The presence of dynamic adjustments triggered by mnemonic load are consistent with previous studies that have found sequential trial performance benefits with higher cognitive demands, including high versus low task difficulty (Fischer et al., 2008) and high versus low perceptual fluency (Dreisbach \& Fischer, 2011). Taken together, there is increasing support for the notion that increased cognitive task 
demands drive dynamic adjustments in cognitive control to facilitate subsequent-trial performance. The high (vs. low) mnemonic load condition, herein, increased current-trial task difficulty. As such, upregulation of cognitive control may have occurred to meet higher cognitive demands. Indeed, the findings that high versus low mnemonic load resulted in subsequent-trial performance benefits over two experiments, herein, replicate the prior findings by Jha and Kiyonaga (2010). Together, these results strengthen the claim that cognitive control is dynamically upregulated in the service of maintaining task performance in the presence of increasing task demands.

The present experiments further extend the findings from the Jha and Kiyonaga (2010) study by demonstrating that in addition to cognitive interference, affective interference can also lead to dynamic adjustments. Whereas negative affect has been previously shown to upregulate cognitive control in the context of response conflict tasks (Zeng et al., 2017), the present experiments demonstrated that negative affective interference in the context of a WM task similarly resulted in subsequent-trial performance improvements. One explanation of these findings is that emotion regulation processes lead to the resolution of negative affective interference by upregulating cognitive resources, perhaps in the service of emotion regulation, to consequently benefit performance. Indeed, previous studies have shown that emotion regulation strategies activate brain regions implicated in the upregulation of cognitive control (Ochsner \& Gross, 2005, 2014). Furthermore, recent findings suggest that response conflict trials employing negative stimuli upregulate cognitive control processes to suppress the processing of task-irrelevant negative stimuli on the subsequent trial, thereby facilitating performance (Steinhauser, Flaisch, Meinzer, \& Schupp, 2016). As such, participants viewing negatively valenced distractors may engage in emotion regulation strategies to increase the availability of cognitive resources. This upregulation of resources may facilitate the suppression of task-irrelevant affective processing in subsequent moments, and thus enhance performance on the following trial.

Broadly, the main findings of the present experiments using affective interference are consistent with those of Jha and Kiyonaga (2010) using cognitive interference. That is, high demands of either mnemonic load or interference were effective in upregulating cognitive control resources to benefit performance on the subsequent trial. In addition, both experiments demonstrated a similar pattern of results as evidenced by comparison of observed means, ORs, and the magnitude of previous-trial effects. Although the presence of a significant interaction for accuracy between previous-trial load and interference varied between experiments, the overall pattern of performance appears to be relatively consistent in both experiments (cf. Figs. 2 and 4), as well as Jha and Kiyonaga (2010).
One potential explanation for the significant interaction observed in Experiment 1 could be related to the high accuracy on trials following the hardest condition (high load/ negative distractors) precluding further improvements. Indeed, the accuracy on trials preceded by high-load and negative-distractor trials (see Table 1$)$ was high $(\sim 91 \%$ in Exp. 1, and $~ 93 \%$ in Exp. 2). Future studies should employ more difficult trial conditions (e.g., high mnemonic load with three items instead of two) to evaluate this possibility.

It is critical to acknowledge the possibility that subsequent performance benefits may not necessarily reflect the upregulation of cognitive control processes (see Egner, 2008). Alternative accounts, including repetition-priming or featurebinding accounts (Hommel et al., 2004; Mayr, Awh, \& Laurey, 2003), suggest that facilitated performance following high-demand trials may occur due to the formation of episodic memories of conflicting stimuli. For example, a high-demand trial may create a memory representation specific to the presentation of the task-relevant and task-irrelevant stimuli. Subsequent presentations of the same stimulus associations activate the memory features, which, in turn, facilitates performance. Another account, the repetition expectancy account, suggests that upregulation of attentional resources is not in the service of resolving conflict, but instead on conscious expectancies regarding the nature of the subsequent trial (Egner, 2008; Gratton et al., 1992). For example, two consecutive presentations of high-load/negative-distractor trials may result in sequential performance benefits due to activation of memory representations just encountered, or due to expectations that a subsequent trial will be of the same type as the previous trial.

However, our results are not wholly consistent with either of these accounts. The repetition-priming account cannot explain our results, because stimuli (memory items or distractors) were randomized across trials, and no stimulus was repeated except for the test items on match trials. In addition, the use of two stimulus categories (e.g., faces and shoes) reduced the chances of repetition priming due to serial presentation of stimuli from one category. Furthermore, the repetition expectancy account cannot explain the observed effects, as trials were pseudo-randomized to avoid consecutive presentations of the same trial type. Thus, our findings cannot be explained by either repetition priming or expectancy effects alone.

Although this has been the first study to investigate dynamic adjustments in response to both mnemonic load and affective interference in a delayed-recognition WM task, the present experiments are not without limitations. The focus herein was specifically on negative interference without examining the impact of positive affective interference. The decision to focus on negative distractors was based on the extant literature on conflict adaptation, suggesting that conflict might serve as a precursor to negative affect and hence might lead to dynamic 
adjustments in cognitive control (see Egner, 2007; Gratton et al., 1992; Hommel et al., 2004; Kerns et al., 2004; Stürmer et al., 2002; Ullsperger et al., 2005; Verbruggen et al., 2006). Indeed, several conflict adaptation studies have investigated negative affect (Dignath et al., 2017; Padmala et al., 2011; van Steenbergen et al., 2009; Zeng et al., 2017). In contrast, fewer studies have examined the impact of positive affect on conflict adaptation, and in the few that have, the results suggest that positive affect might attenuate conflict adaptation (see Dreisbach \& Fischer, 2012a). Similarly, positive distractors presented during the maintenance interval in a delayed-recognition WM task may have a reduced effect on current-trial WM performance when compared to negative distractors (Iordan \& Dolcos, 2017). As such, attenuated dynamic adjustments might be more likely if positive distractors were used. Another potential limitation concerns the mean arousal rating for negative images, which was lower than the normative ratings reported by Lang, Bradley, and Cuthbert (2008). It is possible that our reported effects would not generalize to negative images that were higher in arousal. Future studies should investigate dynamic adjustments in WM across a wider range of affective stimuli varying from positive to negative valence.

The findings of our two experiments suggest that high demands, including mnemonic load and affective interference, trigger the upregulation of cognitive control resources to facilitate subsequent-trial performance. Future studies should increase the number of trials in order to better examine the effects of previous trial demands as a function of current trial demands. Overall, the present findings highlight the ability for multiple aspects of demand to upregulate the availability of resources in the service of adapting to challenging and unpredictable situations.

Acknowledgements The authors thank Ethan Homedi, Shivani Hanchate, Joseph Dunn, and Azin Pooresmaeil for their help with data collection. This work was supported by US Department of Defense, Department of the Army Grant \#W81XWH-11-2-0124, awarded to A.P.J., and by a research grant awarded to J.E.W. by the Department of Psychology at the University of Miami and by Fred C. and Helen Donn Flipse.

\section{References}

Baddeley, A. (1986). Working memory. Oxford, UK: Oxford University Press, Clarendon Press.

Becker, D., Jostmann, N. B., \& Holland, R. W. (2019). Adaptation in conflict: Are conflict-triggered control adjustments protected in the presence of motivational distractors? Cognition and Emotion, 33, 660-672. https://doi.org/10.1080/02699931. 2018.1482825

Botvinick, M. M. (2007). Conflict monitoring and decision making: Reconciling two perspectives on anterior cingulate function. Cognitive, Affective, \& Behavioral Neuroscience, 7, 356-366. https://doi.org/10.3758/CABN.7.4.356
Botvinick, M. M., Braver, T. S., Barch, D. M., Carter, C. S., \& Cohen, J. D. (2001). Conflict monitoring and cognitive control. Psychological Review, 108, 624-652. https://doi.org/10.1037/0033-295X.108.3. 624

Botvinick, M. M., Cohen, J. D., \& Carter, C. S. (2004). Conflict monitoring and anterior cingulate cortex: An update. Trends in Cognitive Sciences, 8, 539-546. https://doi.org/10.1016/j.tics.2004.10.003

Botvinick, M. M., Nystrom, L. E., Fissell, K., Carter, C. S., \& Cohen, J. D. (1999). Conflict monitoring versus selection-for-action in anterior cingulate cortex. Nature, 402, 179-181. https://doi.org/10.1038/ 46035

Braver, T. S., Gray, J. R., \& Burgess, G. C. (2007). Explaining the many varieties of working memory variation: Dual mechanisms of cognitive control. In A. R. A. Conway, C. Jarrold, M. J. Kane, A. Miyake, \& J. N. Towse (Eds.), Variation in working memory (pp. 76-106). New York, NY: Oxford University Press.

Cabeza, R., Dolcos, F., Graham, R., \& Nyberg, L. (2002). Similarities and differences in the neural correlates of episodic memory retrieval and working memory. NeuroImage, 16, 317-330.

Cohen, N., \& Henik, A. (2012). Do irrelevant emotional stimuli impair or improve executive control? Frontiers in Integrative Neuroscience, 6, 33. https://doi.org/10.3389/fnint.2012.00033

Cowan, N. (2016). The many faces of working memory and short-term storage. Psychonomic Bulletin \& Review, 24, 1158-1170. https:// doi.org/10.3758/s13423-016-1191-6

Dignath, D., Janczyk, M., \& Eder, A. B. (2017). Phasic valence and arousal do not influence post-conflict adjustments in the Simon task. Acta Psychologica, 174(Suppl C), 31-39. https://doi.org/10.1016/j. actpsy.2017.01.004

Dolcos, F., Diaz-Granados, P., Wang, L., \& McCarthy, G. (2008). Opposing influences of emotional and non-emotional distractors upon sustained prefrontal cortex activity during a delayed-response working memory task. Neuropsychologia, 46, 326-335. https://doi. org/10.1016/j.neuropsychologia.2007.07.010

Dolcos, F., \& McCarthy, G. (2006). Brain systems mediating cognitive interference by emotional distraction. Journal of Neuroscience, 26, 2072-2079. https://doi.org/10.1523/JNEUROSCI.5042-05.2006

Dolcos, F., Miller, B., Kragel, P., Jha, A., \& McCarthy, G. (2007). Regional brain differences in the effect of distraction during the delay interval of a working memory task. Brain Research, 1152(Suppl C), 171-181. https://doi.org/10.1016/j.brainres.2007. 03.059

Dreisbach, G., \& Fischer, R. (2011). If it's hard to read . . . try harder! Processing fluency as signal for effort adjustments. Psychological Research, 75, 376-383.

Dreisbach, G., \& Fischer, R. (2012a). Conflicts as aversive signals. Brain and Cognition, 78, 94-98. https://doi.org/10.1016/j.bandc.2011.12. 003

Dreisbach, G., \& Fischer, R. (2012b). The role of affect and reward in the conflict-triggered adjustment of cognitive control. Frontiers in Human Neuroscience, 6, 342. https://doi.org/10.3389/fnhum.2012. 00342

Dreisbach, G., \& Fischer, R. (2015). Conflicts as aversive signals for control adaptation. Current Directions in Psychological Science, $24,255-260$.

Egner, T. (2007). Congruency sequence effects and cognitive control. Cognitive, Affective, \& Behavioral Neuroscience, 7, 380-390. https://doi.org/10.3758/CABN.7.4.380

Egner, T. (2008). Multiple conflict-driven control mechanisms in the human brain. Trends in Cognitive Science, 12, 374-380. https:// doi.org/10.1016/j.tics.2008.07.001

Fischer, R., Dreisbach, G., \& Goschke, T. (2008). Context-sensitive adjustments of cognitive control: Conflict-adaptation effects are modulated by processing demands of the ongoing task. Journal of Experimental Psychology: Learning, Memory, and Cognition, 34, 712-718. https://doi.org/10.1037/0278-7393.34.3.712 
Fritz, J., \& Dreisbach, G. (2013). Conflicts as aversive signals: Conflict priming increases negative judgments for neutral stimuli. Cognitive, Affective, \& Behavioral Neuroscience, 13, 311-317. https://doi.org/ 10.3758/s13415-012-0147-1

Fröber, K., Stürmer, B., Frömer, R., \& Dreisbach, G. (2017). The role of affective evaluation in conflict adaptation: An LRP study. Brain and Cognition, 116, 9-16.

Gratton, G., Coles, M. G. H., \& Donchin, E. (1992). Optimizing the use of information: Strategic control of activation of responses. Journal of Experimental Psychology: General, 121, 480-506. https://doi. org/10.1037/0096-3445.121.4.480

Gratton, G., Cooper, P., Fabiani, M., Carter, C.S., \& Karayanidis, F. (2018). Dynamics of cognitive control: Theoretical bases, paradigms, and a view for the future. Psychophysiology, 55, e13016.

Grimm, S., Boesiger, P., Beck, J., Schuepbach, D., Bermpohl, F., Walter, M., ... Northoff, G. (2009). Altered negative BOLD responses in the default-mode network during emotion processing in depressed subjects. Neuropsychopharmacology, 34, 932-843.

Hamilton, J. P., \& Gotlib, I. H. (2008). Neural substrates of increased memory sensitivity for negative stimuli in major depression. Biological Psychiatry, 63, 1155-1162.

Hommel, B., Proctor, R. W., \& Vu, K.-P. L. (2004). A feature-integration account of sequential effects in the Simon task. Psychological Research, 68, 1-17. https://doi.org/10.1007/s00426-003-0132-y

Iordan, A. D., \& Dolcos, F. (2017). Brain activity and network interactions linked to valence-related differences in the impact of emotional distraction. Cerebral Cortex, 27, 731-749.

Jha, A. P., Fabian, S. A., \& Aguirre, G. K. (2004). The role of prefrontal cortex in resolving distractor interference. Cognitive, Affective, \& Behavioral Neuroscience, 4, 517-527. https://doi.org/10.3758/ CABN.4.4.517

Jha, A. P., \& Kiyonaga, A. (2010). Working-memory-triggered dynamic adjustments in cognitive control. Journal of Experimental Psychology: Learning, Memory, and Cognition, 36, 1036-1042. https://doi.org/10.1037/a0019337

Jha, A. P., \& McCarthy, G. (2000). The influence of memory load upon delay-interval activity in a working-memory task: An event-related functional MRI study. Journal of Cognitive Neuroscience, 12(Suppl 2), 90-105. https://doi.org/10.1162/089892900564091

Jha, A. P., Witkin, J. E., Morrison, A. B., Rostrup, N., \& Stanley, E. (2017). Short-form mindfulness training protects against working memory degradation over high-demand intervals. Journal of Cognitive Enhancement, 1, 154-171.

Kerns, J. G., Cohen, J. D., MacDonald, A. W., III, Cho, R. Y., Stenger, V. A., \& Carter, C. S. (2004). Anterior cingulate conflict monitoring and adjustments in control. Science, 303, 1023-1026. https://doi. org/10.1126/science. 1089910

Lakens, D. (2013). Calculating and reporting effect sizes to facilitate cumulative science: A practical primer for $t$ tests and ANOVAs. Frontiers in Psychology, 4, 863. https://doi.org/10.3389/fpsyg. 2013.00863

Lang, P. J., Bradley, M. M., \& Cuthbert, B. N. (2008). International Affective Picture System (IAPS): Affective ratings of pictures and instruction manual (Technical Report A-8). Gainesville, FL: University of Florida, Center for Research in Psychophysiology.

Mayr, U., Awh, E., \& Laurey, P. (2003). Conflict adaptation effects in the absence of executive control. Nature Neuroscience, 6, 450-452. https://doi.org/10.1038/nn1051

Melcher, T., Born, C., \& Gruber, O. (2011). How negative affect influences neural control processes underlying the resolution of cognitive interference: An event-related fMRI study. Neuroscience Research, $70,415-427$.

Miller, E. K., \& Cohen, J. D. (2001). An integrative theory of prefrontal cortex function. Annual Review of Neuroscience, 24, 167-202. https://doi.org/10.1146/annurev.neuro.24.1.167
Ochsner, K. N., \& Gross, J. J. (2005). The cognitive control of emotion. Trends in Cognitive Sciences, 9, 242-249. https://doi.org/10.1016/j. tics.2005.03.010

Ochsner, K. N., \& Gross, J. J. (2014). The neural bases of emotion and emotion regulation: A valuation perspective. In J. J. Gross \& R. Thompson (Eds.), The handbook of emotion regulation (2nd ed., pp. 23-42). New York, NY: Guilford Press.

Padmala, S., Bauer, A., \& Pessoa, L. (2011). Negative emotion impairs conflict-driven executive control. Frontiers in Psychology, 2, 192. https://doi.org/10.3389/fpsyg.2011.00192

Redick, T. S. (2014). Cognitive control in context: Working memory capacity and proactive control. Acta Psychologica, 145, 1-9. https://doi.org/10.1016/j.actpsy.2013.10.010

Saunders, B., Lin, H., Milyavskaya, M., \& Inzlicht, M. (2017). The emotive nature of conflict monitoring in the medial prefrontal cortex. International Journal of Psychophysiology, 119(Suppl C), 31-40. https://doi.org/10.1016/j.ijpsycho.2017.01.004

Shah, S. G., Klumpp, H., Angstadt, M., Nathan, P. J., \& Phan, K. L. (2009). Amygdala and insula response to emotional images in patients with generalized social anxiety disorder. Journal of Psychiatry and Neuroscience, 34, 296.

Sreenivasan, K. K., \& Jha, A. P. (2007). Selective attention supports working memory maintenance by modulating perceptual processing of distractors. Journal of Cognitive Neuroscience, 19, 32-41. https:// doi.org/10.1162/jocn.2007.19.1.32

Steinhauser, M., Flaisch, T., Meinzer, M., \& Schupp, H. T. (2016). Cognitive control modulates preferential sensory processing of affective stimuli. Neuropsychologia, 91, 435-443.

Stürmer, B., Leuthold, H., Soetens, E., Schröter, H., \& Sommer, W. (2002). Control over location-based response activation in the Simon task: Behavioral and electrophysiological evidence. Journal of Experimental Psychology: Human Perception and Performance, 28, 1345-1363. https://doi.org/10.1037/0096-1523.28.6.1345

Ullsperger, M., Bylsma, L. M., \& Botvinick, M. M. (2005). The conflict adaptation effect: It's not just priming. Cognitive, Affective, \& Behavioral Neuroscience, 5, 467-472. https://doi.org/10.3758/ CABN.5.4.467

van Steenbergen, H., Band, G. P. H., \& Hommel, B. (2009). Reward counteracts conflict adaptation: Evidence for a role of affect in executive control. Psychological Science, 20, 1473-1477. https://doi. org/10.1111/j.1467-9280.2009.02470.x

Verbruggen, F., Notebaert, W., Liefooghe, B., \& Vandierendonck, A. (2006). Stimulus-and response-conflict-induced cognitive control in the flanker task. Psychonomic Bulletin \& Review, 13, 328-333. https://doi.org/10.3758/BF03193852

Woltman, H., Feldstain, A., MacKay, J. C., \& Rocchi, M. (2012). An introduction to hierarchical linear modeling. Tutorials in Quantitative Methods for Psychology, 8, 52-69.

Zeng, Q., Qi, S., Li, M., Yao, S., Ding, C., \& Yang, D. (2017). Enhanced conflict-driven cognitive control by emotional arousal, not by valence. Cognition and Emotion, 31, 1083-1096. https://doi.org/10. 1080/02699931.2016.1189882

Open Practices Statement The data for Experiments 1 and 2 are available at https://osf.io/4jpvz/, and Experiment 2 was preregistered at https://osf. io/v8nm5/. Other materials (IAPS image list, image ratings, and questionnaires) will be available upon reasonable request.

Publisher's note Springer Nature remains neutral with regard to jurisdictional claims in published maps and institutional affiliations. 